\title{
HIGH-SCHOOL ALGEBRA OF THE THEORY OF DICRITICAL DIVISORS: ATYPICAL FIBRES FOR SPECIAL PENCILS AND POLYNOMIALS
}

\author{
E. ARTAL BARTOLO, I. LUENGO, AND A. MELLE-HERNÁNDEZ
}

\begin{abstract}
In this work we got a revival of our discussions about dicriticals with Ram. Dedicated to the memory of S.S. Abhyankar.
\end{abstract}

\begin{abstract}
In this work we deal with dicritical divisors, curvettes and polynomials. These objects have been one of the main research interests of S.S. Abhyankar during his last years. In this work we provide some elementary proofs of some S.S. Abhyankar and I. Luengo results for dicriticals in the framework of formal power series. Based on these ideas we give a constructive way to find the atypical fibres of a special pencil and give bounds for its number, which are sharper than the existing ones. Finally, we answer a question of J. Gwoździewicz finding polynomials that reach his bound.
\end{abstract}

\section{INTRODUCTION}

The study of the topology and geometry of polynomial maps is of great interest in Affine Algebraic Geometry, for instance for the cancellation problem or affine exotic spaces. The Jacobian problem is one of the main open problems in this area. Recently the local theory of algebraic dicritical divisors and curvettes has been developed $([13,8,9])$ and applied to get some control on the fibers of a Jacobian pair. Dicritical divisors have been studied by S.S. Abhyankar either alone, [2, 4, 3, 5, 6, 7], or with co-authors, [8, 9, 11, 10, 12, 13, 14. He has developed an algebraic theory which starts from the geometric intuition coming from analytic geometry and extends the result to the more general setting: starting from $\mathbb{C}\{x, y\}$ he developed (with his collaborators) a general theory valid for general regular local rings.

In this work we want to apply this theory to the study of special pencils, i.e., elements of the quotient field of a regular ring whose denominator is a power of a regular element of the ring. The fundamental reason to study these pencils is that they appear naturally when working with polynomial maps at infinity. Moreover, the strategy to study these pencils is through the resolution of the base points of the pencil where dicriticals appear in a natural way. With their algebraic techniques, several results about dicriticals are proved in [13, 14]: the restriction of the pull-back of the pencil to each dicritical is a polynomial, dicriticals are in one-to-one correspondence with the irreducible factors of the pencil, see $\$ 3$ for details.

The core of the paper is to provide elementary algebraic proofs, valid also in positive characteristic, for rings of power series over a field by high-school algebra methods following the mathematical

Date: August 5, 2014.

2010 Mathematics Subject Classification. 14A05, 14R15.

Key words and phrases. dicritical divisor, special pencil. 
philosophy of S.S Abhyankar. In order to achieve the proof, we proceed with a variation of the Newton-Puiseux process realized by birational transformations, see also [18] for similar approaches. Using Newton polygon techniques we describe a finite recursive argument which presents in an explicit case a toric resolution of the pencil which is combinatorially much less complex than the resolution via standard blow-ups or quadratic transformations. With this method, the dicritical divisors are in bijection with some edges of a sequence of Newton polygons, from which we keep two important data: a 1-variable polynomial coming from the edge and a positive integer which is related to a quotient singularity coming from a toric blowing-up.

We will apply these techniques in order to improve some bounds for the number of atypical values of special pencils given by J. Gwoźdiewicz in [22].

Theorem 1.1. (22]) Let $f(x, y), l(x, y) \in \mathbb{C}\{x, y\}, f(0,0)=l(0,0)=0$, be convergent power series without common factor. Assume that the curve $l(x, y)=0$ is smooth and that the curve $f(x, y)=0$ has $d$ components counted without multiplicities. Then, the pencil $f(x, y)-t l(x, y)^{M}=0$, where $M$ is a positive integer, has at most $d$ nonzero atypical values.

Our main result provides a more accurately defined bound for the number of atypical values for a special pencil which is given by the sum of the number of dicriticals plus the number of non-zero roots of the derivatives of the polynomials associated to the dicriticals, see Theorem 2.11. Moreover, this result is true for formal power series over algebraically closed fields without restrictions on the characteristic (except a mild separability hypothesis), following Abhyankar's style. Example 2.14 shows that our bound is sharp.

This local bound is also extended to the polynomial setting, see also [23]. Since at each base point at infinity the polynomial defines a local special pencil then the number of atypical values at infinity is bounded by the sum of the corresponding local bounds we got in Theorem 2.11. Therefore, as a consequence, an algebraic proof of the next Theorem is given.

Theorem 1.2. ([22]) Assume that the complex algebraic curve $f(x, y)=0$ has $n$ branches at infinity. Then the polynomial $f$ has at most $n$ critical values at infinity different from 0 .

We also provide examples showing that our bound is also sharper than the one of [22, Theorem 1.2]. Notice that Gwoźdiewicz's result is in the same spirit as the following Moh's Theorem [26] as quoted by Ephraim's version [21].

Theorem 2.2. ([21]) Assume that the complex algebraic curve $f(x, y)=0$ has only one branch at infinity. Then $f$ has no critical values at infinity. In particular, all curves $f(x, y)=t$ for $t \in \mathbb{C}$ are equisingular at infinity.

As T.T. Moh pointed out in [26, S.S. Abhyankar gave another proof of this result by applying [15, (3.4)].

The number of branches at infinity is related with the Jacobian problem:

if $f_{1}, f_{2} \in \mathbb{K}[x, y]$, char $(\mathbb{K})=0$, is a Jacobian pair, i.e. its Jacobian determinant is equal to 1 , then $\mathbb{K}\left[f_{1}, f_{2}\right]=\mathbb{K}[x, y]$.

T.T. Moh remarks in [26] that the following Engel's statement was a main tool in W. Engel's attempted proof of the Jacobian conjecture, see [20]: 
For a special member of the pencil $f(x, y)+c=0$, the number of branches at infinity cannot be greater than the corresponding number for the general member.

In 1971 S.S. Abyhankar found a counterexample to Engel's statement.

Abhyankar and Moh, see e.g [1] for details, translated the Jacobian condition into conditions on the resulting special expansions getting the following result:

The Two Point Theorem. ([1]) If $f_{1}$ and $f_{2}$ is a Jacobian pair, then $f_{1}$ and $f_{2}$ have at most two points at infinity. Moreover, it can be deduced that if the Jacobian condition implies that $f_{1}$ and $f_{2}$ have at most one point at infinity then the Jacobian problem has an affirmative answer.

In fact if $f_{1}$ and $f_{2} \in \mathbb{K}[x, y]$ is a Jacobian pair with two points at infinity it follows from H. Żoładek in [29] that $f_{1}$ and $f_{2}$ have some common dicriticals. In fact, not all the dicritical components can be in common because in such a case the degree of the polynomial map from $\mathbb{C}^{2}$ to $\mathbb{C}^{2}$ vanishes, hence the Jacobian is identically zero (private communication to the authors of Pierrette Cassou-Noguès).

As we explain in 4 , the conditions to reach this number of branches at infinity are quite involved (in particular Moh-Ephraim result shows that it is not possible when there is only one branch at infinity). The last part of $\$ 4$ is devoted to construct two examples. Example 4.2 is the polynomial version of Example 2.14. Example 4.1 answers positively the following question proposed by J. Gwoźdiewicz [22].

Question. Does there exist a polynomial $f(x, y)$ with $n$ nonzero critical values at infinity such that the curve $f(x, y)=0$ has $n$ branches at infinity?

Example 4.1 is a polynomial where the generic fiber has two branches at infinity. Following a referee's comment we provide in Example 4.3 a way to construct such examples with an arbitrary number of branches at infinity for the generic fiber.

\section{TORIC-NEWTON TRANSFORMS OF SPECIAL MEROMORPHIC FUNCTIONS}

For convenience we work over an algebraically closed field $\mathbb{K}$. Nevertheless, the results are valid over any field since it is well known that one can get the resolution of the base points of a pencil over a finite extension of the base field $\mathbb{K}$. Let $R=\mathbb{K}[[x, y]]$ be the formal power series ring over $\mathbb{K}$; note that most of the results are also valid for convergent power series in case of complex numbers and some of them will also be valid for more general (almost complete) two-dimensional local rings (without restriction on the characteristic and even in mixed characteristic) especially if they have analytical properties, see [9]. Following Abhyankar we will study regular local rings contained in $L$ (the fraction field of $R$ ) and dominating $R$ though we will replace these rings by their completion for simplicity. We will denote $M(R)$ the maximal ideal of $R$.

A formal power series $p(x, y) \in R$ can be evaluated at the only closed point $0 \in \operatorname{Spec} R$, giving

an element $p(0,0) \in \mathbb{K}$. For an element $r(x, y):=\frac{p(x, y)}{q(x, y)}$ the evaluation at 0 can be defined on $\mathbb{P}_{\mathbb{K}}^{1}=\mathbb{K} \cup\{\infty\}$, with one important exception. If $p, q \in M(R)$ are coprime, then $r(0)$ is not defined, it is undetermined. It is also useful to treat $r$ as the pencil of curves $\left\{C_{t}: p=t q\right\}$, for $t \in \mathbb{K} \cup\{\infty\}$ having 0 as base point.

It is well-known that one can eliminate this indetermination via a birational map $\pi: S \rightarrow \operatorname{Spec}(R)$, which is the composition of a sequence of closed points blow-ups, also called quadratic transformations, 
such that $\pi^{*}(r): S \rightarrow \mathbb{P}_{\mathbb{K}}^{1}$ is a well defined morphism. This means that from the point of view of pencils, the strict transforms of the curves $C_{t}$ are disjoint.

Let $E=\pi^{-1}(0)$ be the exceptional divisor of the map $\pi$, with irreducible components $E_{1}, \ldots, E_{s}$. A divisor $E_{i} \subset E$ is called dicritical (or some authors called them horizontal) if $\left.\pi^{*}(r)\right|_{E_{i}}$ is not a constant map, that is $\pi^{*}(r)\left(E_{i}\right)=\mathbb{P}_{\mathbb{K}}^{1}$.

Let us define

$$
\left.P(x, y, T):=p(x, y)-T q(x, y)=\sum_{i, j} A_{i, j} x^{i} y^{j} \in \mathbb{K}(T)[[x, y]] \text { ( } T \text { an indeterminate }\right) .
$$

We have two main interests: To study the curve $\tilde{C}$ given by $P \in \mathbb{K}(T)[[x, y]]$ and to study the curves $C_{t}=\{P(x, y, t)=0\}$ for $t \in \mathbb{K}$, both generic and atypical.

Definition 1.1. The Newton polygon $\operatorname{NP}(r)$ of $r$ is the Newton polygon of $P \in \mathbb{K}(T)[[x, y]]$ i.e. the compact faces of the convex closure of $\operatorname{NR}(P):=\operatorname{Supp}(P)+\mathbb{N}^{2} \subset \mathbb{N}^{2} \subset \mathbb{R}^{2}$.

We are interested in giving several algebraic characterizations of dicritical divisors in a particular class of pencils, specially important for polynomial maps.

Definition 1.2. A meromorphic germ $r \in L$ (or its corresponding pencil) is called special if $r(x, y)=$ $\frac{p(x, y)}{x^{c} U(x, y)}$ for some local parameters $x, y \in R, c>0$ and a unit $U(x, y) \in \mathbb{K}[[x, y]]$ (we always assume that $x$ does not divide $p(x, y))$.

Remark 1.3. Since $x$ does not divide $p$, the $y$-order $d$ of $p(x, y)$ is well-defined, i.e. the unique positive integer such that $p(0, y)$ is a series of order $d$.

Example 1.4. The pencil

$$
p_{x}(x, z, T)=\left(x^{3}-z^{5}\right)^{2}-x^{6}+x\left(x^{5}-z^{2}\right)^{5}+5 x z^{7}\left(x-\frac{3}{4} z^{2}\right)-T z^{11}
$$

is special in $\mathbb{K}(T)[[z, x]]$.

These pencils are called Ephraim pencils in [22], based on [21]. It was shown in [13, Theorem A] that for a special pencil $r$, the restriction of the pull-back $\pi^{*}(r)$ to any dicritical divisor is a polynomial, for arbitrary two dimensional local regular rings, not necessarily equicharacteristic. In this paper an elementary proof of this result for $R=\mathbb{K}[[x, y]]$ is given; the tools used in the proof detect the so-called atypical fibers of the pencil which are also studied in this work.

From now on we assume that $r \in L$ is special. We are going to give a recursive method to solve a special pencil $r$ by means of toric transformations and translations associated to $\mathrm{NP}(r)$.

We introduce some notation. Fix an edge $\ell$ of $\mathrm{NP}(r)$ which is contained in the line $n x+m y=\omega$ $\left(m, n \in \mathbb{N}\right.$ coprime). We denote by $\omega_{\ell}$ the weight $\omega_{\ell}(i, j):=n i+m j$. This edge supports a $\omega_{\ell}$-quasihomogeneous polynomial of degree $\omega$

$$
P_{\ell}(x, y)=\sum_{\omega_{\ell}(i, j)=\omega} A_{i, j} x^{i} y^{j}=x^{u} y^{v} q_{\ell}\left(x^{m}, y^{n}\right),
$$


where $q_{\ell}\left(s_{1}, s_{2}\right) \in \mathbb{K}[T]\left[s_{1}, s_{2}\right]$ is a homogeneous polynomial of degree $d_{\ell}$ with at least two monomials and coprime with $s_{1} s_{2}$. Note that

$$
P(x, y, T)=P_{\ell}(x, y)+\text { monomials with } \omega_{\ell} \text {-degree }>\omega .
$$

The coefficients of $q_{\ell}\left(s_{1}, s_{2}\right)$ are in $\mathbb{K}$ with only one eventual exception: if $v=0$ and $u=c$, i.e., the vertex $(c, 0)$ is in $\ell \subset \mathrm{NP}(r)$. Bezout identity allows to choose

$$
a, b \in \mathbb{Z}_{>0} \text { such that } b n-a m=1 .
$$

Notation 1.5. The coprime weights $(n, m)$ will be denoted if necessary as $\left(n_{\ell}, m_{\ell}\right)$; we will refer to $n$ as the $v$-ratio and $m$ as the $h$-ratio of the edge $\ell$.

The following concept appears also in [18].

Definition 1.6. An edge $\ell$ of $\mathrm{NP}(r)$ is called a dicritical edge if $(c, 0)$ is a vertex of $\ell$

Remark 1.7. We assume that if $(n, m)=(1, m)$ then $P_{\ell}$ is not proportional to $\left(y-A x^{m}\right)^{e}, A \in \mathbb{K}$. If it is the case, the change of variables $y=y_{1}+A x^{m}$ makes the edge $\ell$ disappear. The polygon $\operatorname{NP}(r)$ has at most one dicritical edge.

Example 1.8. Let us consider $p_{x}$ as in Example 1.4. Its Newton polygon is in Figure 1. There is only one edge $\ell$ and $P_{\ell}=\left(x^{3}-z^{5}\right)^{2}$ ( $x$ plays the role of $y$, we keep these variables for further use in 44 ). The edge is not dicritical.

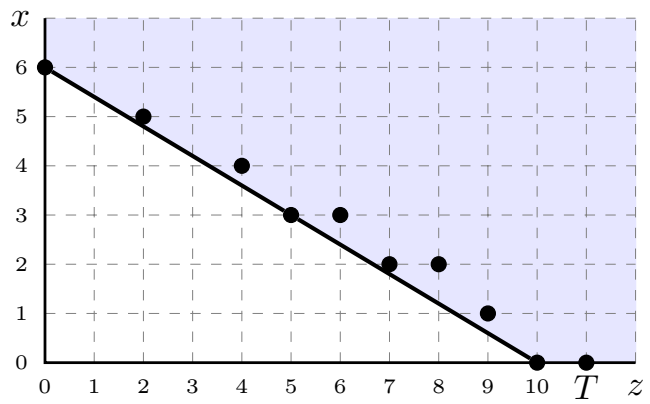

Figure 1. Newton polygon of $p_{x}$

Proposition 1.9. Assume that $\ell$ is not a dicritical edge. The monomial transformation

$$
\varphi_{M}\left(x_{1}, y_{1}\right):=\left(x_{1}^{n} y_{1}^{a}, x_{1}^{m} y_{1}^{b}\right), \quad \text { see (1.2), }
$$

is birational (i.e. it is a composition of quadratic transformations) and the polynomial $P_{\ell}$ is transformed as

$$
P_{\ell}\left(x_{1}^{n} y_{1}^{a}, x_{1}^{m} y_{1}^{b}\right)=\beta x_{1}^{\omega} y_{1}^{a u+b v+a m d_{\ell}} q_{\ell}\left(1, y_{1}\right)
$$


Proof. Note that

$$
P_{\ell}\left(x_{1}^{n} y_{1}^{a}, x_{1}^{m} y_{1}^{b}\right)=\beta x_{1}^{n u+m v} y_{1}^{a u+b v} q_{\ell}\left(x_{1}^{m n} y_{1}^{a m}, x_{1}^{m n} y_{1}^{b n}\right)=\beta x_{1}^{\omega} y_{1}^{a u+b v+a m d_{\ell}} q_{\ell}\left(1, y_{1}\right) .
$$

We use that $\omega=n u+m v+m n d_{\ell}, b n-a m=1$, and the fact that $q_{\ell}$ is homogeneous of degree $d_{\ell}$.

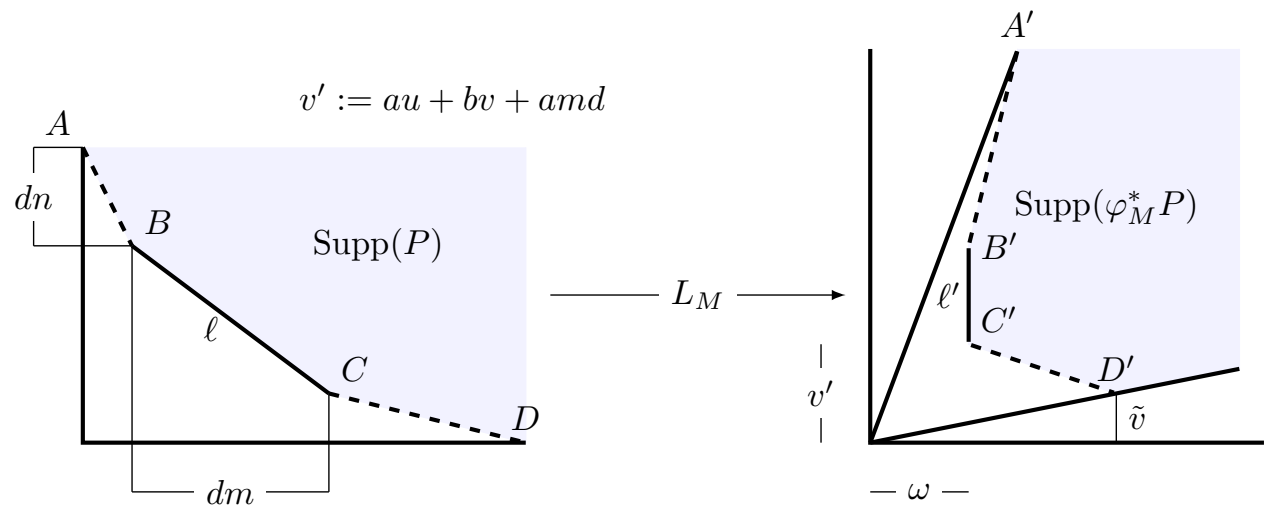

Figure 2. $L_{M}$ for the edge $B C$.

This means that the image of the $\mathrm{NP}(r)$ by the affinity

$$
L_{M}: \mathbb{Z}^{2} \rightarrow \mathbb{Z}^{2},\left(\begin{array}{l}
u \\
v
\end{array}\right) \mapsto\left(\begin{array}{cc}
n & m \\
a & b
\end{array}\right)\left(\begin{array}{l}
u \\
v
\end{array}\right)
$$

has a vertical edge and $\operatorname{Supp}\left(\varphi_{M}^{*} P\right)$, where $\varphi_{M}^{*} P=P\left(x_{1}^{n} y_{1}^{a}, x_{1}^{m} y_{1}^{b}, T\right) \in \mathbb{K}(T)\left[\left[x_{1}, y_{1}\right]\right]$, is contained in $L_{M}(\mathrm{NR}(P))$, see Figure 2, Let us factor

$$
q_{\ell}\left(s_{1}, s_{2}\right)=\beta \prod_{j=1}^{e}\left(s_{2}-\alpha_{j} s_{1}\right)^{m_{j}}, \quad \beta, \alpha_{j} \in \mathbb{K} \backslash\{0\}, \quad m_{j}>0, \text { i.e., } d_{\ell}=\sum_{j=1}^{e} m_{j} .
$$

Definition 1.10. For $\ell$ a non-dicritical edge and $\alpha_{j}$ a root of $q_{\ell}(1, s)$, the toric-Newton transformation associated to $\left(\ell, \alpha_{j}\right)$ is the toric transformation $\varphi_{M}$ followed by the translation $y_{1}=\bar{y}_{1}+\alpha_{j}$.

Definition 1.11. The strict transform $P_{\ell, \alpha_{j}}\left(x_{1}, \bar{y}_{1}, T\right)$ of $P$ by the toric-Newton transformation associated to $\left(\ell, \alpha_{j}\right)$ is

$$
P_{\ell, \alpha_{j}}\left(x_{1}, \bar{y}_{1}, T\right)=\frac{P\left(x_{1}^{n}\left(\bar{y}_{1}+\alpha_{j}\right)^{a}, x_{1}^{m}\left(\bar{y}_{1}+\alpha_{j}\right)^{b}, T\right)}{x_{1}^{\omega}\left(\bar{y}_{1}+\alpha_{j}\right)^{\tilde{v}}} .
$$

where $\tilde{v} \leq a u+b v+a m d$ is the minimum of the powers of $y_{1}$ which appear from the pull-back by $\varphi_{M}$.

Example 1.12. Let us study the strict transform for the toric-Newton transformation of Example 1.4 The Newton polygon of this strict transform is shown in Figure 3a, the quasihomogeneous polynomial is $\left(x_{1}-\frac{5}{2} z_{1}\right)^{2}$ and we are in the situation of Remark 1.7. We perform the translation and we obtain a special pencil whose Newton polygon, in Figure $3 \mathrm{~b}$ has only one edge and it is dicritical since the quasihomogeneous polynomial is $x_{2}^{2}-\left(T+\frac{5}{8}\right) z^{3}$. 


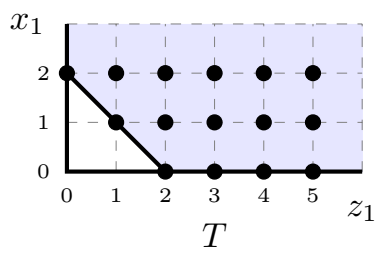

(a)

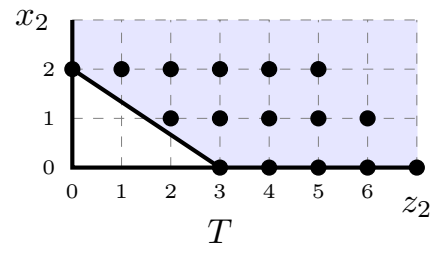

(b)

Figure 3

Proposition 1.13. The strict transform $P_{\ell, \alpha_{j}}\left(x_{1}, \bar{y}_{1}, T\right)$ is a special pencil in $\mathbb{K}\left[\left[x_{1}, \bar{y}_{1}\right]\right]$ such that its $\bar{y}_{1}$-order is $m_{j}$.

Proof. The part of the strict transform corresponding to $P_{\ell}$ is

$$
\beta \bar{y}_{1}^{m_{j}} \prod_{k \neq j}\left(\bar{y}_{1}+\alpha_{j}-\alpha_{k}\right)^{m_{k}} \text {. }
$$

The rest of the strict transform is divided by $x_{1}$. The monomial $T x^{c} U(x, y)$ is transformed into

$$
T x^{n c-\omega}\left(\alpha_{j}+\bar{y}_{1}\right)^{a c-(a u+b v+a m d)} U\left(x_{1}^{n}\left(\bar{y}_{1}+\alpha_{j}\right)^{a}, x_{1}^{m}\left(\bar{y}_{1}+\alpha_{j}\right)^{b}\right)
$$

and the result follows.

We will study later what to do if $\ell$ is a dicritical edge. Because of Proposition 1.13, this process can be also applied to the strict transforms of $P$ by the toric-Newton transformations.

Definition 1.14. The toric-Newton process of $P$ is the sequence of special pencils obtained by applying toric-Newton transformations recursively. The tree of Newton polygons of $P$ is the family of all Newton polygons in the toric-Newton process. An edge of such a Newton polygon is called a dicritical edge if it is at the bottom of the polygon and the coefficient for $(*, 0)$ depends on $T$.

Proposition 1.15. The toric-Newton process is finite.

Proof. Note that the $y$-order of the special pencils decreases unless we are in the situation of Remark 1.7 Since the pencils are special only a finite number of translations may arise until we reach the $T$ monomial. Note that while the term $T x^{c}$ is not present in $N P(r)$ one is following the resolution (of one branch) of the fibre $p(x, y)=0$. This means that after a finite number of toric maps and translations we arrive to a point $Q$ where the branch is non-singular and eventually non-reduced. Then the local equation of the total transform of $P$ is $h^{k}\left(x_{1}, y_{1}\right) u\left(x_{1}, y_{1}\right)+T x_{1}^{e_{1}}$ with $u(0,0) \neq 0$ and $h\left(x_{1}, y_{1}\right)=\left(y_{1}+\ldots\right)$. It is now clear we can make a change of coordinates $y_{1}=\bar{y}+a_{1} x_{1}$ such that $\left.\bar{h}\left(x_{1}, \bar{y}\right)\right)=\bar{y}+a_{e+1} x^{e+1}$

Remark 1.16. Note that this is the case for the pencil in Example 1.4 


\section{DiCRItiCAL EDGES}

Let us study now what happens with dicritical edges. We start with a simple proof of [13, Theorem A] when the regular local ring is a formal power series ring.

Proposition 2.1. Let $P(x, y, T):=p(x, y)-T x^{c} U(x, y)$ be a special pencil, then at each dicritical divisor $E$ the function $\pi^{*}(r(x, y))_{\left.\right|_{E}}$ is a polynomial.

Proof. The previous process allows to resolve the base points of the pencil by toric maps and translations and moreover pencils arising at the process are still special. Let us study what happens at a dicritical edge $\ell$. We keep the notation of (1.1) and we get that

$$
q_{\ell}(1, s)=a_{0} s^{d_{\ell}}+a_{1} s^{d_{\ell}-1}+\cdots+a_{d_{\ell}-1} s-\left(T-a_{d_{\ell}}\right)
$$

where $a_{j} \in \mathbb{K}$. We denote again $\pi\left(x_{1}, y_{1}\right)=\left(x_{1}^{n} y_{1}^{a}, x_{1}^{m} y_{1}^{b}\right)$ the toric transformation associated to $\ell$. Then

$$
P_{\ell}\left(x_{1}^{n} y_{1}^{a}, x_{1}^{m} y_{1}^{b}\right)=x_{1}^{\omega} y_{1}^{\tilde{v}}\left(q_{\ell}\left(1, y_{1}\right)+x_{1} G\left(x_{1}, y_{1}\right)\right),
$$

and $x_{1}=0$ is the equation of $E$ and $G\left(x_{1}, y_{1}\right)$ is some series. Notice that

$$
\frac{\pi^{*}(p)}{\pi^{*}\left(x^{c} U(x, y)\right)}=\frac{x_{1}^{\omega} y_{1}^{\tilde{v}}\left(q_{\ell}\left(1, y_{1}\right)+x_{1} G\left(x_{1}, y_{1}\right)\right)}{x_{1}^{\omega} y_{1}^{\tilde{v}}\left(U(0,0)+x_{1} H\left(x_{1}, y_{1}\right)\right)}=q_{\ell}\left(1, y_{1}\right)+x_{1} G\left(x_{1}, y_{1}\right)
$$

where $U(0,0) \neq 0$ and $H\left(x_{1}, y_{1}\right)$ is some series. Restricting to $x_{1}=0$ we obtain the desired result.

The computations above also prove that the corresponding polynomial map $q_{E}: E \rightarrow \mathbb{P}^{1}$, where $q_{E}(z):=q_{\ell}(1, z)-T$, has degree $d_{E}:=d_{\ell}$.

It is not hard to check that the dicritical divisors of $r$ are in one to one correspondence with the dicritical edges of $\mathrm{NP}(r)$ and its transforms. We study now the toric-Newton transformations for

dicritical edges. Note that the toric part behaves as in the non-dicritical case, as shown in the proof of Proposition 2.1. but the translation part depends on the particular values of $t$. Moreover, separability properties of the polynomial $q_{E}(z)$ have a strong influence on the behavior of the fibers of the pencil near the dicritical $E$.

Proposition 2.2. Let $P(x, y, T)$ be a special pencil as above and let $E$ be a dicritical divisor of $r$ associated to a dicritical edge $\ell$ of the toric-Newton process of $P$. Assume that $q_{E}(z)$ is a separable polynomial, i.e its derivative is not identically zero.

Let $A_{E}^{*}:=\left\{q_{E}(\alpha) \mid q_{E}^{\prime}(\alpha)=0\right\}$ and let $t_{0, E}:=q_{E}(0)$. Then, the strict transform of the germ of the curve $p(x, y)-t x^{c} U(x, y)$ contains exactly $d_{E}$ non-singular transversal curvettes meeting at $d_{E}$ distinct points of $E$, in the following cases:

(1) If $t \notin A_{E}^{*}$ and $t \neq t_{0, E}$.

(2) If $t=t_{0, E}, t \notin A_{E}^{*}$ and $n=1$.

Proof. We start with the first case. Since $t \notin A_{E}^{*}$ and the polynomial $q_{E}(z)$ is separable, we have that $\operatorname{gcd}\left(q_{E}(z)-t, q_{E}^{\prime}(z)\right)=1$ and all the roots of $q_{E}(z)-t$ are simple roots, i.e.:

$$
q_{E}(z)-t=\prod_{i=1}^{d_{E}}\left(z-\alpha_{i}\right), \quad \alpha_{i} \neq \alpha_{j}, \text { if } i \neq j .
$$


Hence, the quasi-homogeneous polynomial associated to the edge $\ell$ for the suitable strict transform of $P(x, y, t)=0$ is

$$
\prod_{i=1}^{d_{E}}\left(y_{1}^{n}-\alpha_{i} x_{1}^{m}\right)
$$

Since $\alpha_{i} \neq 0$ and since $t \neq t_{0, E}$, all the above factors look similar. Hence if we consider the (non trivial) translation $y_{1}=\bar{y}_{1}+\alpha_{i}$

$$
q_{E}\left(\bar{y}_{1}+\alpha_{i}\right)-t=b_{0} \bar{y}_{1}^{d_{E}}+b_{1} \bar{y}_{1}^{d_{E}-1} \ldots+b_{d_{E}-1} \bar{y}_{1}, \quad b_{d_{E}-1} \neq 0 .
$$

If we compose the toric map of the proof of Proposition 2.1 with the above translation, we obtain then, up to terms of higher degree, that the strict transform is written as

$$
b_{0} \bar{y}_{1}^{d_{E}}+b_{1} \bar{y}_{1}^{d_{E}-1} \ldots+b_{d_{E}-1} \bar{y}_{1}+x_{1}(\ldots)
$$

and one gets $d_{E}$ non-singular curves intersecting transversally the dicritical divisor $E:\left\{x_{1}=0\right\}$ at different points.

If $t=t_{0, E}$ is not a root of $q_{E}^{\prime}(z)$ and $n=1$, though the Newton polygon is changing, the factor corresponding to $\alpha_{i}=0$ is again a curvette.

Remark 2.3. With this method, along the exceptional dicritical divisor there will be no base points of the pull-back of the pencil. By this process we get a log-canonical resolution (with quotient singularities) the base points of the pencil. Since at each step we perform toric quadratic transformations we must be careful with the behavior when no translation is needed.

From now on we assume that the map $q_{E}(z)$ is separable, i.e. either $\operatorname{char}(\mathbb{K})=0$ or $\operatorname{char}(\mathbb{K})=p$ and $q_{E}^{\prime}(z) \neq 0$.

Definition 2.4. A value $t \in \mathbb{K}$ is called a typical value for $P(x, y, T)$ at $E$ if the strict transform of the curve $P(t, x, y)$ has exactly $d_{E}$ non-singular branches (curvettes) intersecting $E$ and is called an atypical value for $P(x, y, T)$ at $E$ otherwise.

If $t \in \mathbb{K}$ is a typical value for $P(x, y, T)$ at all dicritical divisors $E$ then $t \in \mathbb{K}$ will be called a typical value for $P(x, y, T)$, and an atypical one otherwise.

Example 2.5. In Figure 3b, we have the Newton polygon of the unique dicritical edge for $p_{x}$ in Example 1.4. If we fix $t=t_{0, E}=-\frac{5}{8}$, the vertex $(0,3)$ disappears. The corresponding Newton polygon is in Figure 4 Since the general fiber is an ordinary cusp and for $t_{0, E}$ we have a tacnode, we conclude that

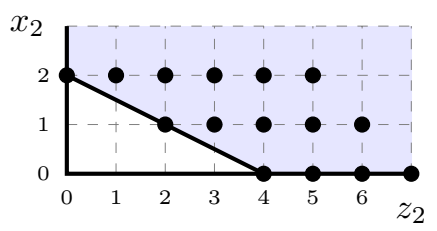

Figure 4. Final Newton polygon for the special fiber

this value is atypical at $E$. 
Remark 2.6. In $\operatorname{char}(\mathbb{K})=0$ this definition is equivalent to the standard definition, see, for instance, the first definition in [24, Section 3]. Note that the cases $(i)$ and (iii) in that definition are not possible for special pencils: $(i)$ in this case is only valid for $t_{0}=q_{E}(0)$ and (iii) is not possible because the first time ones gets a dicritical divisor the linear system has no base points.

We are going to prove a sort of reciprocal of Proposition 2.2 .

Theorem 2.7. Let $P(x, y, T)$ be a special pencil as in Proposition 2.2. Then

(1) If $t \in A_{E}^{*}$ then $t$ is an atypical value for $P(x, y, T)$ at $E$.

(2) If $n>1$ ( $n$ the $v$-ratio) then $t_{0, E}$ is atypical at $E$ regardless the value of $q_{\ell}^{\prime}\left(t_{0, E}\right)$.

Remark 2.8. From the interpretation of dicriticals of Lê-Weber, the case $n>1$ corresponds exactly with the dicriticals which admit a bamboo, see [24], which will be called dicriticals with bamboo.

Proof. For the proof of (1), we follow the ideas in Proposition 2.2. Let $\alpha_{i}$ be a multiple root of $q_{E}(s)-t$. In (2.2), the condition $b_{d_{E}-1} \neq 0$ fails and the corresponding point cannot be a curvette.

For (2), the Newton polygon of $P\left(x, y, t_{0, E}\right)$ has a bottom edge which is non parallel to $\ell$ and of height $n>1$, so there are some branches of this curve which do not meet $E$, see Figure 5 for a typical behavior of Newton polygons.
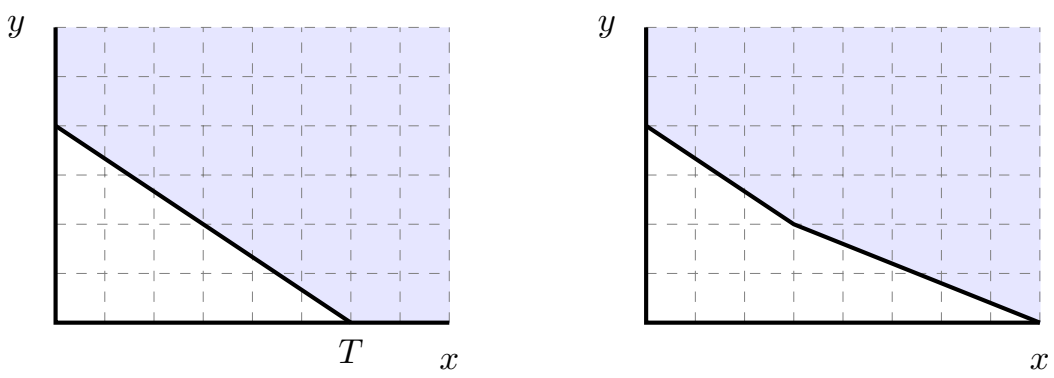

Figure 5. Left-hand-side polygon for generic $T$, right-hand-side for $t_{0, E}$.

Example 2.9. Let us describe some examples.

(1) Consider the special pencil $P(x, y, T)=y^{4}+y^{2} x^{3}+y x^{7}+x^{12}-T x^{6}$, see $\mathrm{NP}(P)$ in Figure6a, The edge $\ell=[(0,4),(6,0)]$ is a dicritical edge such that $P_{\ell}(x, y)=y^{4}+y^{2} x^{3}-T x^{6}, q_{E}(z)=z^{2}+z-T$ and $q_{E}(z)$ is separable. Since the v-ratio $n$ equals $2>1, t=0$ is an atypical value, see its Newton polygon in Figure 6b, On the other side $-\frac{1}{2}$ is the only root of $q_{\ell}^{\prime}$ and then $t=-\frac{1}{4}$ is the other atypical value at $E$, see the Newton polygon after the toric-Newton transformation in Figure 6c. In this case a generic fibre has two branches at $E$ while there are 3 branches for $t=0$ and only one branch for $t=-\frac{1}{4}$.

(2) For the special pencil $P(x, y, T)=y^{3}+y^{2} x-x^{4}-T x^{3}$ the edge $\ell=[(0,3),(3,0)]$ is dicritical and $q_{E}(z)=z^{3}+z^{2}-T$, see $\mathrm{NP}(P)$ in Figure 7a, The derivative has two roots $0,-\frac{2}{3}$, and then $0, \frac{4}{27}$ are the atypical values. Since the v-ratio is $1, t=0$ is atypical only for being a critical 


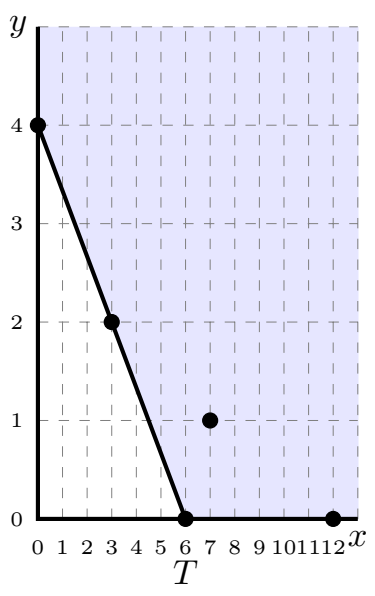

(a)

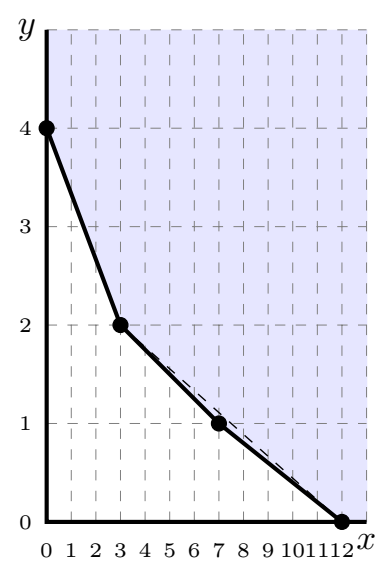

(b)

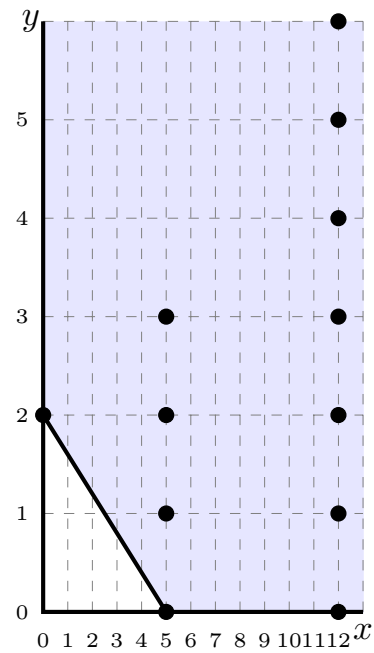

(c)

Figure 6

value of $q_{E}$, see its Newton polygon in Figure $7 \mathrm{~b}$. In order to study the fiber for $t=\frac{4}{27}$, we can check that the quasi-homogeneous polynomial has one simple root and one double root. It is enough to study what happens on the double root; instead of the toric-Newton transformation we can do the change $y=y_{1}-\frac{2}{3} x$, and we obtain again the Newton polygon of Figure $7 \mathrm{~b}$, All the typical fibres have 3 branches while the atypical ones have 2 branches.

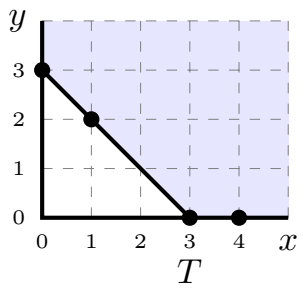

(a)

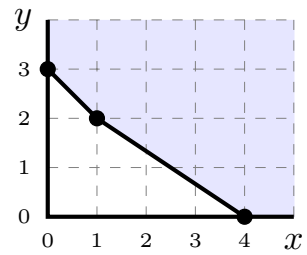

(b)

Figure 7

(3) For the special pencil $P(x, y, T)=y^{3}+y x^{2}-x^{4}-T x^{3}$, the value $t=0$ is typical at the unique dicritical, even if the Newton polygons do not coincide, see Figure 8 .

Remark 2.10. Note that Proposition 2.2 and Theorem 2.7 gives a complete characterization of atypical values of a special pencil in terms of the polynomials $q_{E}(z)$ if they are separable. In the inseparable case the atypical values cannot be computed just from $q_{E}$ as the following examples, in char $(\mathbb{K})=p$, show: 


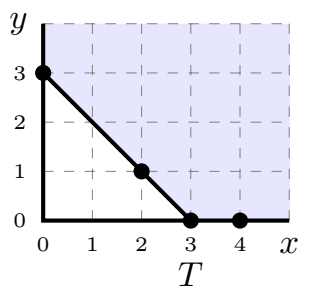

(a)

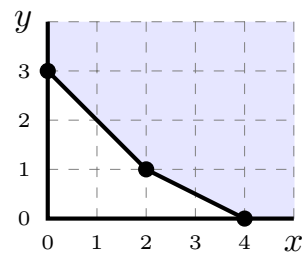

(b)

Figure 8

(a) $y^{p}+x^{p+1}-T x^{p}, t=0$ behaves as the other values of $\mathbb{K}$.

(b) $y^{p}+y^{2} x^{p-1}+T x^{p}, t=0$ does not behave as the other values of $\mathbb{K}$.

In both cases the generic members of the pencil have the singularity type of $y^{p}+x^{p+1}$. In particular it is not a curvette, as curvettes are smooth, and following our definition all values would be atypical. A natural extension of our definition to the non-separable case would imply that $t=0$ is typical for (a) and atypical for (b). See [25] for a more complete description of pencils in positive characteristic.

In the separable case, we can recover algebraically the results of [22]. More precisely it is possible to recover the number of atypical fibers only in terms of the Newton polygons. The type of the atypical fibers needs the part behind the Newton polygons, but for the number, these Newton polygons are enough, compare with Remark 2.10.

We would like to estimate the number of atypical values at a dicritical. Let us collect the relevant information from the Newton process. We have $E_{1}, \ldots, E_{r}$ dicriticals coming from dicritical edges $\ell^{1}, \ldots, \ell^{r}$, each one carries a polynomial $q_{i}(z):=q_{E_{i}}(z)$ of degree $d_{i}$ and from the weight $\omega_{\ell^{i}}$ we keep the number $n_{i}$. The separability hypothesis asserts that $q_{i}(z)$ is separable.

Theorem 2.11. Let $P(x, y, T)$ be a special pencil satisfying the separability hypothesis. Let $E$ be a dicritical and let $n$ be its v-ratio. Let $A_{E}$ be the set $\left\{q_{E}(\alpha) \mid q_{E}^{\prime}(\alpha)=0\right\}$. Then, the set of atypical values for $P(x, y, T)$ at $E$ is

$$
\begin{cases}A_{E} \cup\left\{q_{E}(0)\right\} & \text { if } n>1 \\ A_{E} & \text { if } n=1\end{cases}
$$

In particular, the number of atypical values for $P(x, y, T)$ at $E$ is at most

$$
M_{E}:=\#\left\{\text { non-zero roots of } q_{E}^{\prime}\right\}+1,
$$

and the number of atypical values for $P(x, y, T)$ is at most $\sum_{E \text { dicritical }} M_{E}$.

Proof. This is a direct consequence of Theorem 2.7.

The following result is an easy consequnece of Theorem 2.11

Corollary 2.12 (Gwoździewicz [22]). Let $P(x, y, T)$ be a special pencil.

(1) If $E$ is a dicritical divisor of degree $d_{E}$, then there are at most $d_{E}$ atypical values at $E$. 
(2) If there is a value $t_{0}$ such that $C_{t_{0}}^{\mathrm{red}}$ has $r$ branches at a dicritical divisor $E$ then there are at most $r$ atypical values at $E$ (besides eventually $t_{0}$ ).

(3) The number of atypical values of the pencil is bounded by $\min \left(\nu^{\text {gen }}, \nu^{\min }+1\right)$, where $\nu^{\text {gen }}$ is the number of branches of the generic value and $\nu^{\mathrm{min}}$ is the minimal number of branches of the fibers.

Remark 2.13. In order to reach the bound $\nu^{\text {gen }}$, the following conditions must happen. For every dicritical $E$, one has $n>1, q_{E}^{\prime}\left(t_{0, E}\right) \neq 0, q_{E}^{\prime}$ has simple roots, and these roots have distinct values by $q_{E}$. Moreover, the sets of atypical values for each dicritical are pairwise disjoint.

Example 2.14. Let us consider the special pencil

$$
P(x, y, T)=y^{4}-2 x^{2} y^{2}+\left(y^{2}-x^{2}\right) y x^{2}+x^{7}-T x^{4}
$$

which, for all $t \in \mathbb{K}$ has 4 branches; the bound proposed in Corollary 2.12, see 22], for the number of atypical values is at most 4 . Let us compute the bound of Theorem 2.11. The unique edge $\ell$ of the Newton polygon is dicritical and for its dicritical $E$ we have $q_{E}(z)=z^{4}-2 z^{2}-T$. The roots of $q_{E}^{\prime}(z)$ are $\alpha=0,1,-1$, hence the bound equals 3 . Since $q_{E}(0)=0$ and $q_{E}(1)=q_{E}(-1)=1$, there are exactly two atypical values, $t=0,1$. Figure 9a shows $\operatorname{NP}(P(x, y, T))$, while Figure 9b shows $\operatorname{NP}(p(x, y))$. Note that Figure $9 \mathrm{~b}$ shows also $\mathrm{NP}(p(x, y \pm x)-1)$.

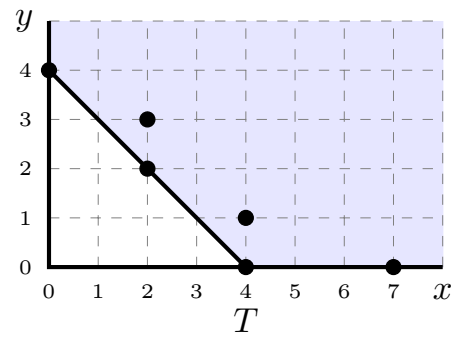

(a)

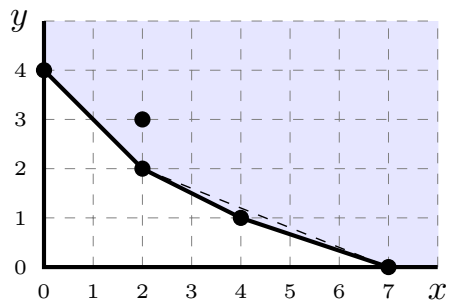

(b)

Figure 9

\section{FaCtors of a SPECial Pencil over $\mathbb{K}(T)$}

Let us interpret a result of [14] in this language, always in the special case of power series, namely that the dicritical divisors of $r$ are in one-to-one correspondence with the factors of $P(x, y, T)$ in $\mathbb{K}(T)[[x, y]]$.

Fix a dicritical edge and keep the notations of Proposition 2.1.

Proposition 3.1. Let $\ell$ be a dicritical edge of the $\mathrm{NP}(r)$ corresponding to a dicritical divisor $E$. Then there exists an irreducible factor $Q_{\ell}(x, y, T) \in \mathbb{K}(T)[[x]][y] \subset \mathbb{K}(T)[[x, y]]$ of an element $P(x, y, T)$ such that its weighted initial form for $\omega_{\ell}$ equals $q_{\ell}\left(x^{m_{\ell}}, y^{n_{\ell}}\right)$. 
Proof. Note first that using Weierstraß Preparation Theorem, $P(x, y, T)$ can be decomposed as a product of a unit and a Weierstraß polynomial in $y$ (recall that $P$ is $y$-regular of order $d$ ). We apply the version of Hensel's Lemma in $\mathrm{A}$ to this Weierstraß polynomial and the result follows.

Remark 3.2. Instead of using Hensel's Lemma one can follow the ideas in [17, Section 2]

If the dicritical edge $\ell$ is in another special pencil $r_{1}$ of the toric-Newton process with coordinates $\left(x_{1}, \bar{y}_{1}\right)$, then Proposition 3.1 allow us to construct an irreducible factor $\tilde{Q}_{\ell}\left(x_{1}, \bar{y}_{1}\right)$ of $r_{1}$ in $\mathbb{K}(T)\left[\left[x_{1}\right]\right]\left[y_{1}\right]$; this factor is $\bar{y}_{1}$-regular of order $d_{\ell}$. Let us see the effect of the inverse of the toric-Newton transformation in this element which produced $r_{1}$. The toric Newton transformation has two parts; the inverse of the translation is $\bar{y}_{1} \mapsto \bar{y}_{1}+\alpha_{j}=y_{1}$ while $\varphi_{M}^{-1}\left(x_{1}, y_{1}\right)=\left(x_{1}^{b} y_{1}^{-m}, x_{1}^{-a} y_{1}^{n}\right)=(\bar{x}, \bar{y})$. Hence, the inverse of the toric-Newton transformation will is

$$
\left(x_{1}, \bar{y}_{1}\right) \mapsto\left(x_{1}^{b}\left(\bar{y}_{1}+\alpha_{j}\right)^{-m}, x_{1}^{-a}\left(\bar{y}_{1}+\alpha_{j}\right)^{n}\right)=(\bar{x}, \bar{y}) .
$$

Taking out denominators we obtain $\bar{Q}_{\ell}(\bar{x}, \bar{y})$ which is a divisor of the special pencil $\bar{P}(\bar{x}, \bar{y}, T)$ at this level. It is not hard to see that $\bar{Q}_{\ell}(\bar{x}, \bar{y})$ is $\bar{y}$-regular of order $n d_{\ell}$. The contribution of this factor to the $\bar{y}$-degree is the expected one. We continue till we arrive to the first level; at each step the degree on the $y$-coordinate is multiplied by the corrsponding v-ratio. The final pull-back $Q_{\ell}$ of $\tilde{Q}_{\ell}$ (taking out denominators) to $\mathbb{K}(T)[[x, y]]$ is an irreducible factor of $P(x, y, T)$.

Let $\ell^{1}, \ldots, \ell^{s}$ be the dicritical edges of the toric-Newton process. For each dicritical edge $\ell^{i}$ we consider the sequence of v-ratios $n_{1}^{i}, \ldots, n_{h_{i}}^{i}\left(h_{i}\right.$ is the number of steps till $\ell^{i}$ appear) and its degree $d_{\ell^{i}}$. The factor $Q_{\ell^{i}}$ has $y$-order

$$
d_{i}:=d_{\ell_{i}} \cdot \prod_{j=1}^{h_{i}} n_{j}^{i}
$$

If $d=\operatorname{ord}_{y}(P)$, note that $d=\sum_{j=1}^{s} d^{j}$ and we conclude the next Theorem, see [14] in more generality.

Theorem 3.3. Let $P(x, y, T)$ be a special pencil. Then there is a one-to-one correspondence between dicritical edges of the pencil and irreducible factors of $P \in \mathbb{K}[T][[x, y]]$. By this correspondence to an edge $\ell^{j}$ we associate the factor $Q_{\ell^{j}}$.

For typical $t \in \mathbb{K}$ the irreducible components of $\tilde{C}_{t}:=\{P(x, y, t)=0\}$, i.e. $\operatorname{Spec}(R /(P(x, y, t)))$, are in one-to-one correspondence with the factors of $P(x, y, T)$ in $\overline{\mathbb{K}(T)}[[x, y]]$ and the factors corresponding to a given factor in $\mathbb{K}[T][[x, y]$ are the curvettes of the corresponding dicritical (as many as the degree).

\section{Special pencils, polynomials and atypical fibers}

In this section we recall the well-known relationship between special pencils and polynomials. The polynomial $f(x, y) \in \mathbb{K}[x, y], D:=\operatorname{deg} f$, defines a polynomial map $f: \mathbb{A}_{\mathbb{K}}^{2} \rightarrow \mathbb{A}_{\mathbb{K}}^{1}$, where $\mathbb{A}^{j}:=\mathbb{A}_{\mathbb{K}}^{j}$ is the affine space of dimension $j$ over $\mathbb{K}$. We consider $(x, y)$ the affine coordinates of $\mathbb{A}^{2}$ and $[X: Y: Z]$ the homogeneous coordinates of $\mathbb{P}^{2}:=\mathbb{P}_{\mathbb{K}}^{2}$ with the inclusion $(x, y) \hookrightarrow[x: y: 1]$. Let us consider the rational extension of $f$ to a map $\tilde{f}: \mathbb{P}^{2} \rightarrow \mathbb{P}^{1} \equiv \mathbb{K} \cup\{\infty\}$. If $f(x, y)=\sum_{j=0}^{D} f_{j}(x, y)$ is the decomposition in homogeneous components then

$$
B:=\left\{[u: v: 0] \mid f_{D}(u, v)=0\right\}
$$


is the set of base points of $\tilde{f}$. At every base point $P_{0} \in B$ (at the line at infinity) the corresponding pencil is an special pencil.

Assume that $P_{0}:=[1: 0: 0]$ is one of these points. In the affine chart $X \neq 0$ (with affine coordinates $y, z)$ this map looks like

$$
\frac{f_{y}(y, z)}{z^{D}}, \quad f_{y}(y, z):=z^{D} f\left(\frac{1}{z}, \frac{y}{z}\right)
$$

and the fibers of $\tilde{f}$ near $P_{0}$ are of the form $f_{y}(y, z)-t z^{D}=0$, for $t \in \mathbb{K} \cup\{\infty\}$, hence a special pencil.

By definition the dicriticals of the polynomial $f$ at infinity are the dicriticals of the corresponding special pencils at all base points $P_{0} \in B$. We define accordingly the atypical values at infinity at a dicritical of the polynomial $f$, see also [19].

In [22], Gwoździewicz finds that the number of atypical values at infinity of a polynomial is bounded above by the minimum of the two following numbers:

- The number $\nu_{\infty}^{\text {gen }}$ of the branches at infinity of a generic fiber.

- The number $\nu_{\infty}^{\min }+1$ where $\nu_{\infty}^{\min }$ is the minimal number of branches at infinity for any fiber.

Therefore an algebraic proof of these results follows immediately from our algebraic proof of Corollary 2.12,

In the same work, Gwoździewicz asked if it is possible to reach the bound $\nu_{\infty}^{\operatorname{gen}}\left(\right.$ or $\left.\nu_{\infty}^{\min }+1\right)$. As we have observed in Remark 2.13, to reach this bound imposes strong conditions on the special pencils over all the dicriticals $E$ :

- $n_{E}>1$.

- $q_{E}^{\prime}$ must have simple roots.

- $q_{E}$ must pairwise separate the values of 0 and the roots of $q_{E}^{\prime}$.

- The sets of atypical values are disjoint for any pair of dicriticals.

When we deal with polynomials the last condition must be applied to any dicritical at infinity. Besides this difficulty the geometry of the polynomials imposes more difficulties to find an example reaching the bound.

Namely, no polynomial with only one dicritical reaches the bound. Assume for simplicity that the polynomial is primitive. Then, the only dicritical is of degree 1, see e.g. [16]. Hence, by [27] all the fibers have only one branch at infinity and by [21, there is no atypical value at infinity. It is not hard to find polynomials with two dicriticals $E_{1}, E_{2}$ both of multiplicity one but $n_{i}>1$. These polynomials have two branches at infinity and one atypical value for each dicritical. The problem is that most obvious examples satisfy that the set of atypical values is the same for both dicriticals.

Gwoździewicz's question. Does there exist a polynomial $f(x, y)$ with $n$ nonzero critical values at infinity such that the curve $f(x, y)=0$ has $n$ branches at infinity?

Example 4.1. No polynomial of degree $\leq 10$ and two dicriticals reaches the bound. The polynomial

$$
p(x, y)=x^{6} y^{5}-5 x^{5} y^{4}+10 x^{4} y^{3}-2 x^{3} y^{3}-10 x^{3} y^{2}+5 x^{2} y^{2}+5 x^{2} y-\frac{15}{4} x y-x+y
$$


does. We will show that this polynomial $p(x, y)$ has two non-zero critical values at infinity and the curve $p(x, y)=0$ has two branches at infinity. This polynomial can be written as

$$
p(x, y)=\left(x^{3} y^{2}-1\right)^{2} y+(x y-1)^{5} x-x^{6} y^{5}+5 x y\left(x y-\frac{3}{4}\right) .
$$

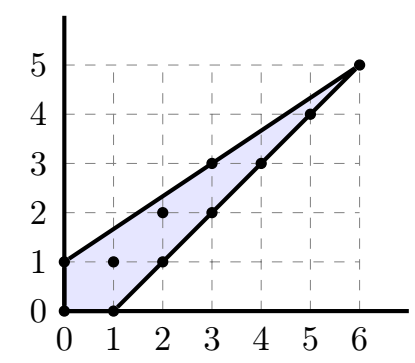

(a) Newton polygon of $p$

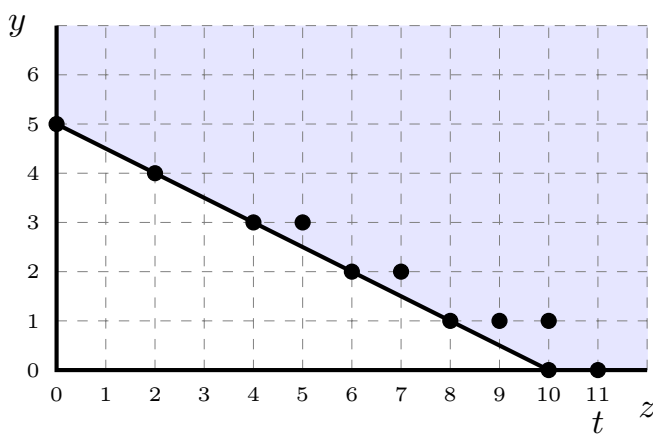

(b) Newton polygon of $p_{y}$

Figure 10

In order to obtain the resolution of the polynomial we have to study the special pencils located at the two points at infinity of $p$. The first one is given by

$$
p_{x}(x, z)=\left(x^{3}-z^{5}\right)^{2}+\cdots-t z^{11}
$$

and it is the one in Example 1.4 (see also Example 2.5). We have seen that it has only one dicritical which is of degree one and v-ratio 2 . There is only one atypical value for this dicritical, namely $t=-\frac{5}{8}$.

Let us study now the special pencil associated to the other point at infinity:

$$
p_{y}(y, z)=\left(y-z^{2}\right)^{5}+\cdots-t z^{11}
$$

We are in the situation of Remark 1.7, hence we perform a translation as a change of variables, $y=y_{1}+z_{1}^{2}, z=z_{1}$. In Figure 11a we see the new Newton polygon where the coefficient of $z^{11}$ equals $-\left(t+\frac{3}{4}\right)$. The Newton polygon for $t=-\frac{3}{4}$ is in Figure $11 \mathrm{~b}$ Hence, there is one atypical value for this polynomial associated to this dicritical.

Then, the two atypical values for each dicritical are different and the polynomial $p$ reaches the bound: as many non-zero atypical fibers at infinity as branches at infinity for the fiber $p(x, y)=0$. The two atypical fibers at infinity have three branches. The polynomial $p$ has only one (affine) singular fiber $p^{-1}\left(-\frac{20}{27}\right)$ which has an ordinary double point at $\left(-900,-\frac{4}{3375}\right)$.

Example 4.2. In the same way as in the local case, see Example 2.14 the following polynomial shows that our bounds are better than the ones in [22]. Consider the following polynomial of degree 10 (see 


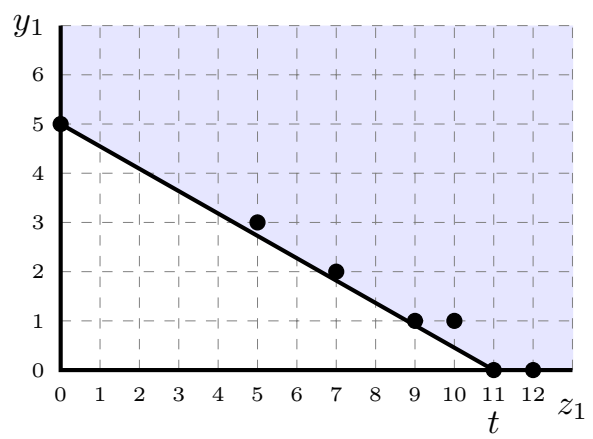

(a)

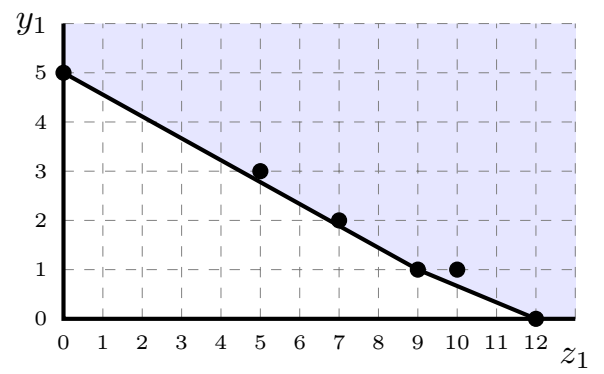

(b)

Figure 11

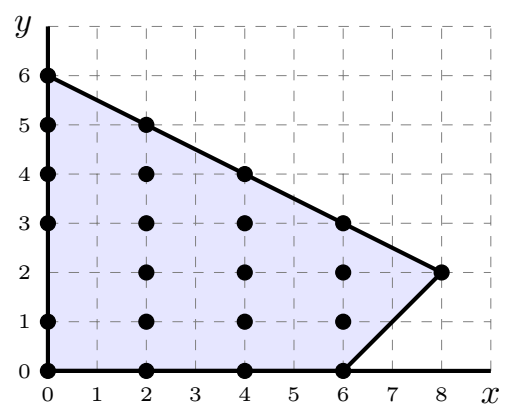

(a) Newton polygon of $f$

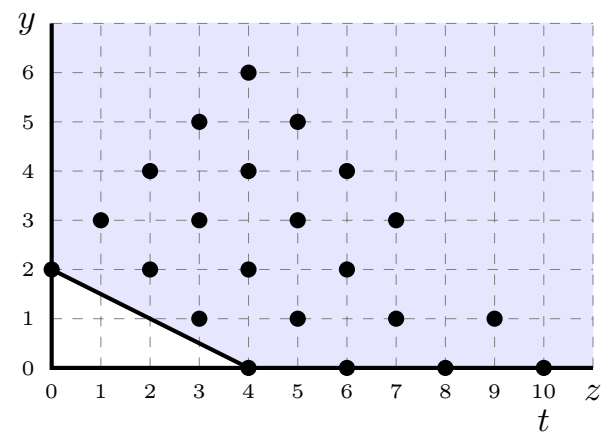

(b) Newton polygon of $f_{y}$

Figure 12

its Newton polygon in Figure 12a):

$$
\begin{gathered}
f(x, y)=y^{6}-4\left(x^{2}+1\right) y^{5}+\left(12 x^{2}+6 x^{4}+\frac{41}{4}\right) y^{4}-\left(4 x^{6}+\frac{25}{2}+12 x^{4}+\frac{99}{4} x^{2}\right) y^{3} \\
+\left(x^{8}+4 x^{6}+\frac{75}{4} x^{4}+\frac{59}{4} x^{2}\right) y^{2}+\left(-\frac{17}{4} x^{6}+\frac{75}{4} x^{2}+4 x^{4}+\frac{25}{4}\right) y \\
-\frac{25}{2} x^{2}-\frac{25}{4} x^{6}-\frac{71}{4} x^{4} .
\end{gathered}
$$

This polynomial has two points at infinity, that is $P_{0}=[1: 0: 0]$ and $P_{1}=[0: 1: 0]$ Thus the corresponding special pencil at $P_{0}$ is given by

$$
\begin{gathered}
f_{y}(z, y)-T z^{10}=y^{6} z^{4}-4 y^{5} z^{3}-4 y^{5} z^{5}+12 y^{4} z^{4}+6 y^{4} z^{2}+\frac{41}{4} y^{4} z^{6}-4 y^{3} z-\frac{25}{2} y^{3} z^{7} \\
-12 y^{3} z^{3}-\frac{99}{4} y^{3} z^{5}+4 y^{2} z^{2}+\frac{75}{4} y^{2} z^{4}+\frac{59}{4} y^{2} z^{6}+y^{2}-\frac{17}{4} y z^{3} \\
+\frac{75}{4} y z^{7}+4 y z^{5}+\frac{25}{4} y z^{9}-\frac{25}{2} z^{8}-\frac{25}{4} z^{4}-\frac{71}{4} z^{6}-T z^{10}
\end{gathered}
$$


Let us see that this special pencil has 2 branches for all $t \in \mathbb{K}$ and it has two dicriticals $E_{1}$ and $E_{2}$ of degree 1. Its Newton polygon (see Figure $12 \mathrm{~b}$ ) has only one edge $\ell$ which is not dicritical and such that

$$
P_{\ell}=y^{2}-\frac{25}{4} z^{4}=\frac{\left(2 y-5 z^{2}\right)\left(2 y+5 z^{2}\right)}{4} .
$$

Thus $q_{\ell}$ has degree 2 and two simple roots $\pm \frac{5}{2}$. Making the toric-Newton transformation associated to each root $\left(\ell, \pm \frac{5}{2}\right)$ one gets two dicriticals, each one of degree 1 (which are sections with no bamboo). Moreover, these two dicriticals have no atypical value associated.

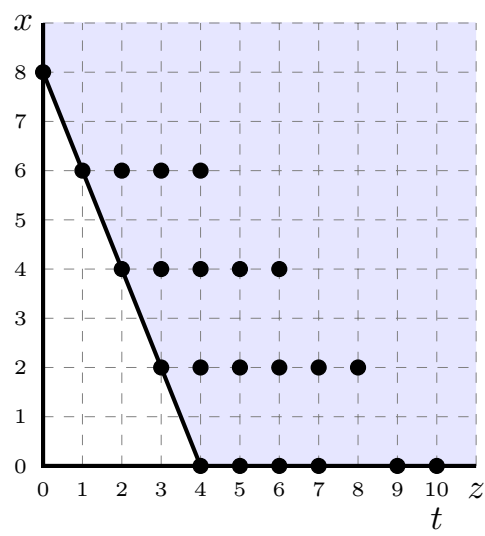

(a) Newton polygon of $f_{x}$

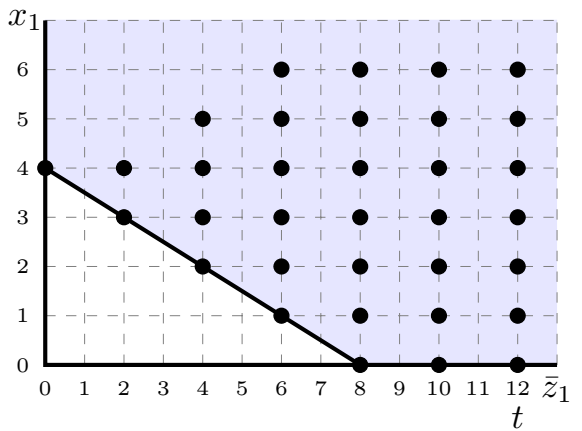

(b) Newton polygon of $f_{x, 1}$

Figure 13

The other special pencil at $P_{1}$ is given by

$$
\begin{gathered}
f_{x}(z, x)-T z^{10}=-\frac{25}{4} z^{4} x^{6}-\frac{17}{4} z^{3} x^{6}+4 z^{2} x^{6}-4 x^{6} z-12 z^{3} x^{4}+6 x^{4} z^{2} \\
-\frac{71}{4} z^{6} x^{4}+4 z^{5} x^{4}+\frac{75}{4} z^{4} x^{4}-\frac{25}{2} z^{8} x^{2}+\frac{75}{4} z^{7} x^{2}+\frac{59}{4} z^{6} x^{2}-\frac{99}{4} z^{5} x^{2} \\
+12 x^{2} z^{4}-4 x^{2} z^{3}+z^{4}-4 z^{5}+\frac{41}{4} z^{6}-\frac{25}{2} z^{7}+\frac{25}{4} z^{9}+x^{8}-T z^{10} .
\end{gathered}
$$

Let us check that this special pencil has 4 branches for all $t \in \mathbb{K}$ and one dicritical $E$. Its Newton polygon is in Figure 13a, there is only one edge $\ell$, which is not dicritical and the quasihomogenous polynomial associated to the edge is $P_{\ell}=\left(x^{2}-z\right)^{4}$. We need only one toric-Newton transformation at this stage:

$$
\varphi_{M}\left(z_{1}, x_{1}\right)=\left(z_{1}^{2} x_{1}, z_{1} x_{1}\right), \quad x_{1} \mapsto \bar{x}_{1}+1
$$

The Newton polygon of the strict transform $f_{x, 1}\left(z_{1}, \bar{x}_{1}\right)$ is in Figure 13b. We have only one edge $\ell_{1}$, which is non-dicritical with $P_{\ell_{1}}=\left(\bar{x}_{1}+z_{1}^{2}\right)^{4}$. If we perform the translation of Remark 1.7 we obtain a new special pencil $f_{x, 2}\left(z_{2}, x_{2}\right)$. The Newton polygon is in Figure 14. We have only one edge $\ell_{2}$, which is dicritical, since

$$
P_{\ell_{2}}=x_{2}^{4}-2 x_{2}^{2} z_{2}^{5}+(1-T) z_{2}^{12}
$$


i.e., its v-ratio equals $2, q_{\ell}(z)=z^{4}-2 z^{2}+1-T$ and $q_{E}(z)=z^{4}-2 z^{2}+1$. The roots of $q_{E}^{\prime}(z)$ are $\alpha=0,1,-1$, hence the bound equals 3. Since $q_{E}(0)=0$ and $q_{E}(1)=q_{E}(-1)=1$, there are exactly two atypical values, $t=0,1$.

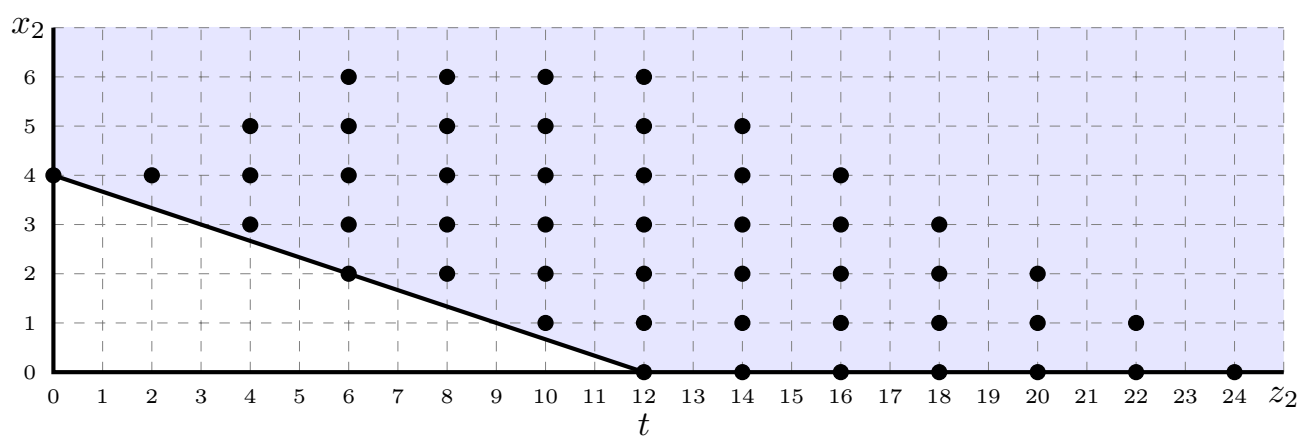

Figure 14. Newton polygon of $f_{x, 2}$

Example 4.3. The referee asked whether Gwoździewicz's question has an affirmative answer for other positive integer $n \geq 3$. In this example we provide a polynomial family which confirms the required positive answer.

For any $d$, we consider two monic polynomials $q(t), Q(t) \in \mathbb{K}[t]$ of degrees $2 d$ and $2 d+1$, respectively, such that:

(C1) $\operatorname{deg}(Q(t)-t q(t)) \leq d$.

(C2) $q(t)=\prod_{j=1}^{m}\left(t-a_{j}\right)^{m_{j}}, \sum_{j=1}^{m} m_{j}=2 d, m_{j} \geq 2$.

(C3) $Q(t)=\prod_{j=1}^{n}\left(t-b_{j}\right)^{n_{j}}, \sum_{j=1}^{n} n_{j}=2 d+1, n_{j} \geq 2$.

Let $f(x, y)$ be the polynomial

$$
f(x, y)=(y+1)(x q(x y)+(y+1) Q(x y)) .
$$

Its Newton polygon has four edges whose vertices are given by

$$
[(0,0),(0,1),(2 d+1,2 d+2),(2 d+1,2 d),(1,0)] .
$$

Let $\ell_{1}=[(0,1),(2 d+1,2 d+2)], \ell_{2}=[(1,0),(2 d+1,2 d)]$ and $\ell_{3}=[(2 d+1,2 d),(2 d+1,2 d+2)]$ be the edges not passing through the origin.

The support polynomial $f_{\ell_{1}}$ is $y Q(x y)$. Because of condition (C3) one can see that each root $b_{j}$ induces a dicritical section with bamboo, producing exactly one atypical value.

The support polynomial $f_{\ell_{2}}$ is $x q(x y)$. As above, condition $(\mathrm{C} 2)$ implies that each root $a_{j}$ induces a dicritical section with bamboo, producing exactly one atypical value.

The support polynomial of the vertical edge $\ell_{3}$ is $f_{\ell_{3}}=x^{2 d+1} y^{2 d}(y+1)^{2}$. The condition (C1) implies that the translation $y=y_{1}-1$ produces a new edge $\ell_{3}^{\prime}=[(0,0),(2 d+1,2)]$. Hence $\ell_{3}^{\prime}$ is a dicritical edge with bamboo $\left(v_{\ell_{3}^{\prime}}=2\right)$ and only one atypical value. 
Of course the conditions (C1), (C2) and (C3) impose restrictions but one can see that solutions exist and for generic choices, the atypical values for each dicritical are distinct, providing the required affirmative answer.

For example, this is the case if

$$
Q(t)=(t+1)^{2 d+1} \text { and } p(t)=\left(\prod_{j=1}^{d}\left(t-a_{j}\right)\right)^{2}
$$

Then (C1) allows to give the coefficients of $p(t)$. A tedious verification ensures that $f$ has $d+2$ dicritical sections with bamboo, and the generic fiber has $d+2$ branches at infinity, its genus being $d$. The fact that they have $d+2$ different atypical values has been checked for small values of $d(\leq 20)$ with SAGE [28].

Remark 4.4. Note that for $d=1$, we can obtain a polynomial with three branches and degree 7 , while Example 4.1, with two branches, has degree 11. Surprisingly, this is the smallest degree for a two-branch polynomial reaching the bound. Note that all the examples have only dicritical sections.

Example 4.5. Both Examples 4.1 and 4.3 have only dicritical sections. We have found also an example of degree 18, with two dicriticals (with bamboo), one of them $E$ with multiplicity 2, hence having also three branches at infinity for the generic fiber and three atypical values. The fiber corresponding to the value in $A_{E}^{*}$ has only two branches at infinity, i.e., $\nu_{\infty}^{\text {min }}+1=\nu_{\infty}^{\text {gen }}$, check the bounds in Page 15 .

\section{ACKNOWLEDGEMENTS}

First author is partially supported by MTM2010-21740-C02-02; the last two authors are partially supported by the grant MTM2010-21740-C02-01. The authors would like to thank Pierrette CassouNoguès and referees for useful remarks and valuable comments.

\section{Appendix A. Hensel's Lemma}

In order to be clear which flavor of Hensel's Lemma we are going to use, we state and prove the following elementary result

Let $\mathbb{K}$ be a field and fix a weight $\omega(x, y):=n x+m y$ for $n, m \in \mathbb{N}$. Given $0 \neq F \in \mathbb{K}[[x, y]]$, we will consider its decomposition in $\omega$-quasihomogeneous forms

$$
F(x, y)=F_{a+b}(x, y)+F_{a+b+1}(x, y)+\ldots,
$$

where the subindex means the $\omega$-weight

Lemma A.1 (Hensel's Lemma). Asume that $F_{a+b}(x, y)=f_{a}(x, y) g_{b}(x, y), f_{a}, g_{b} \in \mathbb{K}[x, y]$ quasihomogeneous and coprime.h Then, there exist

$$
f, g \in \mathbb{K}[[X, Y]], \quad f=f_{a}+f_{a+1}+\ldots, \quad g=g_{b}+g_{b+1}+\ldots
$$

such that $F=f g$. Moreover if $f_{a}$ is an irreducible polynomial, then $f$ is an irreducible power series. 
Proof. We need to find recursively $\omega$-quasihomogeneous polynomials $f_{a+k}, g_{b+k}, k \in \mathbb{N}$ such that

$$
f_{a}(x, y) g_{b+k}(x, y)+g_{b}(x, y) f_{a+k}(x, y)=F_{a+b+k}^{*}(x, y)
$$

where $g_{b+k}, f_{a+k}$ are the unknowns and $F_{a+b+k}^{*}$ is obtained from $F_{a+b+k}$ and the previous solutions for $k^{\prime}<k$.

Let us decompose the above polynomials (where now the subindex correspond now to the homogeneous degree for the weigh $\omega_{0}$ defined by $\left.n=m=1\right)$ :

$$
\begin{array}{rlrr}
f_{a}(x, y)= & x^{a_{x}} y^{a_{y}} f_{a^{\prime}}\left(x^{m}, y^{n}\right), & a= & n a_{x}+m a_{y}+a^{\prime} m n \\
g_{b}(x, y)= & x^{b_{x}} y^{b_{y}} g_{b^{\prime}}\left(x^{m}, y^{n}\right), & b= & n b_{x}+m b_{y}+b^{\prime} m n \\
f_{a+k}(x, y)= & x^{c_{x}} y^{c_{y}} \tilde{f}_{c}\left(x^{m}, y^{n}\right), & a+k= & n c_{x}+m c_{y}+c m n \\
g_{b+k}(x, y)= & x^{d_{x}} y^{d_{y}} \tilde{g}_{d}\left(x^{m}, y^{n}\right), & b+k= & n d_{x}+m d_{y}+d m n \\
F_{a+b+k}^{*}(x, y)= & x^{e_{x}} y^{e_{y}} \tilde{F}_{e}\left(x^{m}, y^{n}\right), & a+b+k= & n e_{x}+m e_{y}+e m n .
\end{array}
$$

The decompositions of $a, b, c, d, e$ are unique if we assume that the all indices are non-negative, the coefficient of $n$ is less than $m$ and the coefficient of $m$ is less than $n$. We prove it in several steps.

Claim 1. The statement holds for $\omega_{0}$, i.e., the homogeneous case.

It is an immediate consequence of the properties of the resultant.

Claim 2. The statement holds if $f_{a}(x, y)$ is a power of $x$ or $y$.

Assume that $f_{a}$ is a power of $x$. In this case, we have

- $a=n\left(a_{x}+m a^{\prime}\right), 0 \leq a_{x}<m$.

- $g_{b}(0,1) \neq 0$, i.e., $b_{x}=0$.

The following equalities hold:

$$
n\left(a_{x}+d_{x}\right)+m d_{y}+\left(a^{\prime}+d\right) m n=n c_{x}+m\left(b_{y}+d_{y}\right)+\left(b^{\prime}+c\right) m n=n e_{x}+m e_{y}+e m n .
$$

We deduce that $e_{x}=c_{x}=a_{x}+d_{x}-\alpha m, e_{y}=d_{y}=b_{y}+d_{y}-\beta n$, where $\alpha, \beta \in\{0,1\}$ and

$$
e=a^{\prime}+d+\alpha=b^{\prime}+c+\beta .
$$

Equation (A.2) is equivalent to

$$
x^{\alpha+a^{\prime}} \tilde{g}_{d}(x, y)+y^{\beta} \tilde{g}_{b^{\prime}}(x, y) \tilde{f}_{c}(x, y)=\tilde{F}_{e}(x, y),
$$

which follows from Claim 1, and Claim 2 holds.

Claim 3. The statement holds if both $f_{a}$ and $g_{b}$ are coprime with $x, y$.

In this case $a_{x}=a_{y}=b_{x}=b_{y}=0$ and

$$
d_{x}=e_{x}, \quad d_{y}=e_{y}, \quad a^{\prime}+d=b^{\prime}+c=e .
$$

Hence (A.2) is transformed again in its homogeneous version and Claim 3 follows from again from Claim 1. Combining these claims, the statement is proved. 


\section{REFERENCES}

[1] S.S. Abhyankar, Lectures on expansion techniques in algebraic geometry, Tata Institute of Fundamental Research Lectures on Mathematics and Physics, vol. 57, Tata Institute of Fundamental Research, Bombay, 1977, Notes by Balwant Singh.

[2] _ Dicritical divisors and Jacobian problem, Indian J. Pure Appl. Math. 41 (2010), no. 1, 77-97.

[3] _ More about dicriticals, Proc. Amer. Math. Soc. 139 (2011), no. 9, 3083-3097.

[4] - Pillars and towers of quadratic transformations, Proc. Amer. Math. Soc. 139 (2011), no. 9, 3067-3082.

[5] _ Quadratic transforms inside their generic incarnations, Proc. Amer. Math. Soc. 140 (2012), no. 12, 41114126.

[6] Dicriticals of pencils and Dedekind's Gauss lemma, Rev. Mat. Complut. 26 (2013), no. 2, 735-752.

[7] _ Generic incarnations of quadratic transforms, Proc. Amer. Math. Soc. 141 (2013), no. 12, 4103-4117.

[8] S.S. Abhyankar and E. Artal, Algebraic theory of curvettes and dicriticals, Proc. Amer. Math. Soc. 141 (2013), no. $12,4087-4102$.

[9] _ Analytic theory of curvettes and dicriticals, to appear in Rev. Mat. Complut., doi:10.1007/s13163-014-01484, 2014.

[10] S.S. Abhyankar and W.J. Heinzer, Existence of dicritical divisors revisited, Proc. Indian Acad. Sci. Math. Sci. 121 (2011), no. 3, 267-290.

[11] _ Existence of dicritical divisors, Amer. J. Math. 134 (2012), no. 1, 171-192.

[12] L Rees valuations, Proc. Indian Acad. Sci. Math. Sci. 122 (2012), no. 4, 525-546.

[13] S.S. Abhyankar and I. Luengo, Algebraic theory of dicritical divisors, Amer. J. Math. 133 (2011), no. 6, $1713-1732$.

[14] _ Spiders and multiplicity sequences, Proc. Amer. Math. Soc. 141 (2013), no. 12, 4071-4085.

[15] S.S. Abhyankar and T.T. Moh, Newton-Puiseux expansion and generalized Tschirnhausen transformation. I, II, J. Reine Angew. Math. 260 (1973), 47-83; ibid. 261 (1973), 29-54.

[16] E. Artal, Une démonstration géométrique du théorème d'Abhyankar-Moh, J. Reine Angew. Math. 464 (1995), 97-108.

[17] E. Artal, Pi. Cassou-Noguès, I. Luengo, and A. Melle-Hernández, On ע-quasi-ordinary power series: factorization, Newton trees and resultants, Topology of algebraic varieties and singularities, Contemp. Math., vol. 538, Amer. Math. Soc., Providence, RI, 2011, pp. 321-343.

[18] Pi. Cassou-Noguès, Newton trees at infinity of algebraic curves, Affine algebraic geometry, CRM Proc. Lecture Notes, vol. 54, Amer. Math. Soc., Providence, RI, 2011, pp. 1-19.

[19] A.H. Durfee, Five definitions of critical point at infinity, Singularities (Oberwolfach, 1996) (Basel), Progr. Math., no. 162, Birkhäuser, 1998, pp. 345-360.

[20] W. Engel, Ein Satz über ganze Cremona-Transformationen der Ebene, Math. Ann. 130 (1955), 11-19.

[21] R. Ephraim, Special polars and curves with one place at infinity, Singularities, Part 1 (Arcata, Calif., 1981), Proc. Sympos. Pure Math., vol. 40, Amer. Math. Soc., Providence, RI, 1983, pp. 353-359.

[22] J. Gwoździewicz, Ephraim's pencils, Int. Math. Res. Not. IMRN (2013), no. 15, 3371-3385.

[23] Z. Jelonek and M. Tibar, Bifurcation locus and branches at infinity of a polynomial $f: \mathbb{C}^{2} \rightarrow \mathbb{C}$, Preprint available at arXiv:1401.6544v1 [math.AG] .

[24] D.T. Lê and C. Weber, Équisingularité dans les pinceaux de germes de courbes planes et $C^{0}$-suffisance, Enseign. Math. (2) 43 (1997), no. 3-4, 355-380.

[25] A. Melle-Hernández and C.T.C. Wall, Pencils of curves on smooth surfaces, Proc. London Math. Soc. (3) 83 (2001), no. 2, 257-278.

[26] T.T. Moh, On analytic irreducibility at $\infty$ of a pencil of curves, Proc. Amer. Math. Soc. 44 (1974), 22-24.

[27] _ On the Jacobian conjecture and the configurations of roots, J. Reine Angew. Math. 340 (1983), $140-212$.

[28] W.A. Stein et al., Sage Mathematics Software (Version 5.10), The Sage Development Team, 2013, http://www. sagemath.org.

[29] H. Żołądek, An application of Newton-Puiseux charts to the Jacobian problem, Topology 47 (2008), no. 6, 431-469. 
iUma, Departamento de Matemáticas, Facultad de Ciencias, Universidad de Zaragoza, C/ Pedro CerBUNA 12, 50009 ZARAGOZA, SPAIN

E-mail address: artal@unizar.es

iCMAT (CSiC-UAM-UC3M-UCM), Departamento de Álgebra, Facultad de Ciencias Matemáticas, Universidad Complutense, 28040 Madrid, SPAIN

E-mail address: iluengo@mat.ucm.es, amelle@mat.ucm.es 